\title{
Index
}

'1968' 20, 22, 47, 60, 107

Åberg, Carl Johan 116

see also 'quintuplets'

$\mathrm{ABF}$, Arbetarnas bildningsförbund,

Worker's Educational Association 78

active labour market policy 37

see also Rehn-Meidner model

active social policies 36,52

see also productive social policy;

productivism

affluent society 28

see also Galbraith, John Kenneth, The

affluent society

\section{Aftonbladet 69}

Åkerman, Nordal 69

Almedalen, SAP annual economic seminar 132

American capitalism 68 see also Americanisation; poor relief fattigvård; workfare

Americanisation 78

asocial 31

Aspling, Sven 29, 52

see also strong society; Social welfare in evolution, Socialvård i utveckling

Association of Directors of Swedish

Welfare Services, Föreningen Sveriges

socialchefer, FSS 47, 52

ATP, pension reform 30

'battle of the elm trees' 66

Blair-Schröder document 131

Branting, Hjalmar 73
Bretton Woods-system 28

collapse of 96,105

bridge-over policy 96

Brooks-report 85

Brown, Gordon 132

Budget Commission 101

Carlsson, Ingvar 106, 116, 118, freedom of choice 119

Freedom speech 119

Carson, Rachel 66

Silent spring 66

Cassel, Gustaf 7, 13

Socialpolitiken, Social Policy 7

childcare 42, 66, 117

citizen's wage 58

see also Equality Group

class conflict 28

Club of Rome 68

limits to growth, The 68

see also Palme, Olof

Committee on Low Income,

Låginkomstutredningen 46, 47, 49, 50, 51, 56, 70, 97

see also Holmberg, Per

Commons, John 7

community work 82

Coordinated economic policy, Samordnad näringspolitik 37, 38, 39, 70

cost-benefit analysis 100 see also productivity and productivity measures

Crisis Group 112

see also Crisis Programme 
Crisis Programme, A future for Sweden, Framtid för Sverige 1, 107, 112-16 see also modernisers; Kanslihushöger;

Social Democratic Economists; war of the roses

Crosland, Anthony 19

CSA, Swedish Association for Social Work, Centralförbundet för socialt arbete 7

\section{Dagens nyheter 29}

Depression 5, 58, 102, 132

dismantling of the welfare state, social nedrustning, systemskifte 106, 112

economic democracy $69,76,79$ see also wage earner funds

Edberg, Ralf 66

Skuggan av ett moln, The shadow of a cloud efterkrigsprogrammet 66

the Labour movement's post-war programme 30

Eklund, Klas 109, 116, 125

see also 'sixtuplets'

Ely, Richard 7

En demokratisk hushållning, Democratic economic planning 97

equality-efficiency dilemma 10

Equality Group 56, 69, 70, 95 see also Equality report

Equality report, More equality, Ökad jämlikhet 56, 57

Equality report, Welfare and work for all, Välfärd och arbete åt alla 69

Erlander, Tage 29, 31, 32, 40, 132 affluent society, The 40

People working together, Människor $i$ samverkan, 31, 119

politics of progress, The, Framstegens politik 31, 119

society of freedom of choice, The, Valfrihetens samhälle, A 31, 119 see also public sector; rising expectations; strong society

ESO, the Expert Group For Study of the Public Sector, Expertgruppen för studier i offentlig ekonomi 106, 111, 117

Esping-Andersen, Gösta 10 three worlds of welfare capitalism, The 11

European social model 132

Fabianism 7

Fälldin, Torbjörn 101

Fälldin government 101

family policy 10

Feldt, Kjell-Olof 21, 102, 117

freedom of choice 119

see also Ministry of Finance

freedom

of choice 33, 119-21

negative and positive freedom 119

security 33

Freire, Paolo 82

Pedagogy of indignation, Pedagogik för förtryckta 82

Friedman, Milton 106

FNL-movement 22

future programme, The future in the hands of the people, Framtiden i hela folkets händer 118

Galbraith, John Kenneth 33, 40

affluent society, The 33, 40

GDP measure 28, 40, 41, 53, 98, 100

Geijer, Arne 56

gender equality 66

see also childcare

general social policies 5,8 see also Möller, Gustav; universalism

German historical school, Kathedersocialismus 7

Group 822

Hall, Peter A 23

Hall, Stuart 20

Hansson, Per Albin 5, 79 see also people's home (folkhemmet)

Harrington, Michael 49 other America, The 49

Heckscher, Eli 7

Hedborg, Anna 122

Hellström, Mats 95, 102

Holmberg, Bo 117

Holmberg, Per 47, 54, 59, 99 see also Committee on Low Income 
income maintenance 31

see also universalism

industrial policy $89,90-2$

Inghe, Gunnar and Maj 46

Unfinished welfare, Den ofärdiga välfärden 46

Jarl, Stefan 77, 78

dignified life, A, Ett anständigt liv 77, 78

They call us mods, Dom kallar oss mods 77

see also Palme, Olof

Johansson, Bo 110

Johansson, Sten 99, 109

Kanslihushöger 21

modernisers

see also Ministry of Finance

Karleby, Nils 102

Keynes, John Maynard 8

General theory 8

Keynesianism

deficit spending 96, 108

Mitterand-style 17

social 6

Kolk, Jan 110

knowledge groups 23

\section{laissez faire 7}

Långtidsutredning, 5-year prognosis 28, 42

liberals, Liberal party, 8, 33, 34, 132

Limits to growth, the see Club of Rome

Lisbon strategy 131

living standard measure 50, 54, 70, 97

LO, Swedish Trade Union Confederation

$22,37,122$

see also Coordinated economic policy,

Samordnad näringspolitik; Low

wages and welfare, Låglön och

Välfärd; Report on the work

environment; Results and reforms,

Resultat och reformer; Social goals

and planning, Sociala mål $i$

samhällsplaneringen; trade union

movement and the welfare state,

The, fackföreningsrörelsen och

välfärdsstaten

Low-Income study 35

Low wages and welfare, Låglön och välfärd
$50,51,53,54$

Marshall plan 28

Marxism

Austrian 7

critical social democracy 22

Olof Palme 60, 61

radical social work 20

McCracken report 101

Meidner, Rudolf 58, 64, 110

Threatened sector, Hotad sector 111

Miljonprogrammet 67

Ministry of Finance 117, 118 see also Feldt, Kjell-Olof;

Kanslihushöger

Ministry of Public Administration, Civildepartementet 117

mixed economy 9,39

modernisers 21

see also Kanslihushöger; Ministry of

Finance

Möller, Gustav 5, 8, 13, 58

monetarism 14, 101, 106

Mundebo, Ingemar 111

see also Budget Commission

Myrdal, Alva 5, 8, 63, 79, 96

see also Equality Group; Equality report

Myrdal, Gunnar 1, 5, 6, 7, 63, 89, 96, 114, 132

Do social reforms cost money?, Kosta sociala reformer pengar 8

see also population question;

Stockholm school, new

neo-liberalism 14, $20,101,106-7$

New deal 14

see also New Labour

New Labour 12, 13, 18, 132

new public management 14

Nuder, Pär 132

OECD, Organisation of Economic

Cooperation and Development

Brooks-report, The 85

McCracken report 101

Social indicators 98

Offensive for social justice 121

other America, The see Harrington, Michael, The other America 
Palme, Olof 29, 60, 62, 84, 120

196860,61

Åkerman, Nordal 69

Brandt, Willy 68

Club of Rome 68

economists 102

Erlander, Tage 29

A dignified life, Ett anständigt liv 77

growth 67-9

Kreisky, Bruno 68

neo-liberalism 106

people's home 79

social exclusion $67-9,76-80$

Söderström, Herbert 78

third way 68,105

utopia 119

parliamentary election

1976101

$1979106-7$

1982 106-7

Pedagogy of indignation, Pedagogik för

förtryckta 82

see also Freire, Paolo

people's home (folkhemmet) 5, 9, 15, 17,

$29,79,131$

renewing the people's home 118

Persson, Göran 15, 131

Pinochet, Augusto 106

planhushållning, planning 30

Policy Network 132

poor relief, fattigvård 31

American 122

population economics 8

population question 5

see also Myrdal, Gunnar

productive consumption 39

productive social policy $1,6,8$

see also prophylactic; productivism; universalism

productivism 10-12, 37

see also universalism, workfare

productivity and productivity measures

$41,53,98-101,106,108-12,122-3$

see also public sector

programme budgeting, PPB 100

prophylactic 6

see also productive social policy

public good 39, 100

public sector 30,33 inflation 42

private sector 93

productivity and productivity measures

$41,53,98-101,106,108-12,122-3$

programme budgeting PPB 100

public good 39, 100

renewal 118

social balance 39,100

quality of life 70

see also welfare

'quintuplets' 116

see also Åberg, Carl Johan

Reagan, Ronald 106

regional policy 65

rehabilitation 43,52

see also active labour market policy; active social policies; productive social policy; productivism

Rehn, Gösta 58, 125

Rehn-Meidner model 30, 37, 43, 47, 49, 70

renewal 118

Report on the work environment,

Fackföreningsrörelsen och den tekniska utvecklingen 46

Results and reforms, Resultat och reformer 37

rising expectations 33

see also Erlander, Tage

SAF, the Swedish Employers' Federation,

Svenska arbetsgivareföreningen 106, 111

SAMAK, Council of Nordic Trade Unions

110

trade union movement and the public sector, The, Fackföreningsrörelsen och den offentliga sektorn 110

Sassoon, Donald 28

Silent spring 66 see also Carson, Rachel

'sixtuplets' 116 see also Eklund, Klas

Skuggan av ett moln, The shadow of a cloud 66

Edberg, Ralf

Smith, Adam 110 
wealth of nations, The 111

social balance 39,100

see also Galbraith, John Kenneth;

public sector

Social Committee, socialutredningen 80

Social Democratic Economists,

Socialdemokratiska ekonomgruppen,

SDE 107-12

social exclusion $46-9,50-4,67,70-8,84$

see also Palme, Olof; welfare

Social goals and social planning, Sociala

mål $i$ samhällsplaneringen 53

social heritage 50,76

social indicators 98

social insurance reforms 1950s 30

social legislation 195632

see also poor relief

Social Policy Committee 35, 36, 51

Socialpolitiken, Social Policy 7

see also Cassel, Gustaf

Socialtjänstlagen, SoL, Social Welfare Act 1982, 83, 106, 121

Social welfare in evolution, Socialvård $i$ utveckling 72

Söderström, Herbert 78

see also Palme, Olof

SOFI, Institute for Social Research 71, 99

Soviet communism 68

Stockholm school, new 5, 6, 8, 108

see also Depression, Myrdal, Gunnar;

Wigforss, Ernst; Unemployment

Commission

Stockholm school, old 7, 8, 132

see also Cassell, Gustaf; Heckscher, Eli

Sträng, Gunnar 29, 42, 54, 59, 102

strong society $1,28,29,37,46,47,55,57$, $60,66,88,118,121$

supply-side orientation 10,37

see also productivism

Swedish Municipal Workers' Union,

Kommunalarbetareförbundet 110

Swedish Union of Metal Workers,

Metallarbetareförbundet 106

swedosclerosis 101
Tawney, R.H. 7

Thatcher, Margaret 106, 111

Thatcherism 17, 20

third way $1,14,17,18,107,113,121$, 131

British; Anglo-Saxon 2, 11, 17, 131-2

revisionism 17

see also Palme, Olof

Third World 60

Threatened sector, Hotad sector 111

see also Meidner, Rudolf

Timbro 106

Tingsten, Herbert 29

trade union movement and the welfare state, The, Fackföreningsrörelsen och välfärdsstaten 122

trickle-down 28, 40

underclass, social heritage 50, 76

Unemployment Commission 5

Unfinished welfare, Den ofärdiga välfärden 46

see also Inghe, Gunnar and Maj

universalism 2, 11

utopia 119

see also Palme, Olof

Verein fur Sozialpolitik 7

Vietnam war 60

von Hayek, Friedrich 106

wage earner funds $21,69,76,79$

see also economic democracy

Wagner, Adolf 7

war of the roses 116

welfare, välfärd 69, 70, 71, 84, 90, 91, 97

see also quality of life

Wigforss, Ernst 7, 8, 102, 114, 132

Can we afford to work?, Ha vi råd att arbeta? 114

third way 19,114

planning, planhushållning 30

workfare 12, 131

see also productivism 